\title{
An Integrated Double Balanced Mixer on Multilayer Liquid Crystalline Polymer (M-LCP) Based Substrate
}

\author{
Wansuk Yun ${ }^{1}$, Vinu Govind ${ }^{1}$, Sidharth Dalmia ${ }^{2}$, Venky Sundaram ${ }^{1}$, Madhavan Swaminathan ${ }^{1}$, \\ and George E. White ${ }^{2}$ \\ ${ }^{1}$ Georgia Institute of Technology, Electrical and Computer Engineering, Atlanta, GA 30332, U.S.A, 404-385-6417 \\ ${ }^{2}$ Jacket Micro Devices, Suite 213, $755^{\text {th }}$ Street, Altanta, GA 30308, USA, 404-526-6046
}

\begin{abstract}
This paper presents the design of an integrated double balanced mixer on a novel multilayer LCP based substrate. Low-loss lumped and distributed components were realized on a 3-layer LCP stackup, which were then used for the design of novel wideband baluns. A double balanced mixer for WLAN applications was realized using embedded baluns in the substrate. The mixer exhibits 5.1-8.6dB conversion loss over a frequency band of 3.5$5.5 \mathrm{GHz}$.
\end{abstract}

\section{INTRODUCTION}

Increasing system complexity, combined with a drive towards a reduction in size and cost, has powered the need for higher levels of integration in RF front ends. System on package (SOP) has shown promise for supporting the convergence of multiple wireless/wired communication systems, thereby enabling reduced radio form factors. SOP provides functionality in the package through the integration of passives such as inductors [1], capacitors and resistors. Low loss organic materials like Liquid Crystalline Polymer (LCP) allow the implementation of high-Q passives embedded in the packaging substrate, allowing the designer to achieve completely integrated wireless systems [2]. However, multilayer substrates with high-density interconnects are critical in meeting size targets.

Fig1 shows a multiband RF front end architecture. This paper presents a RF mixer using embedded passives.

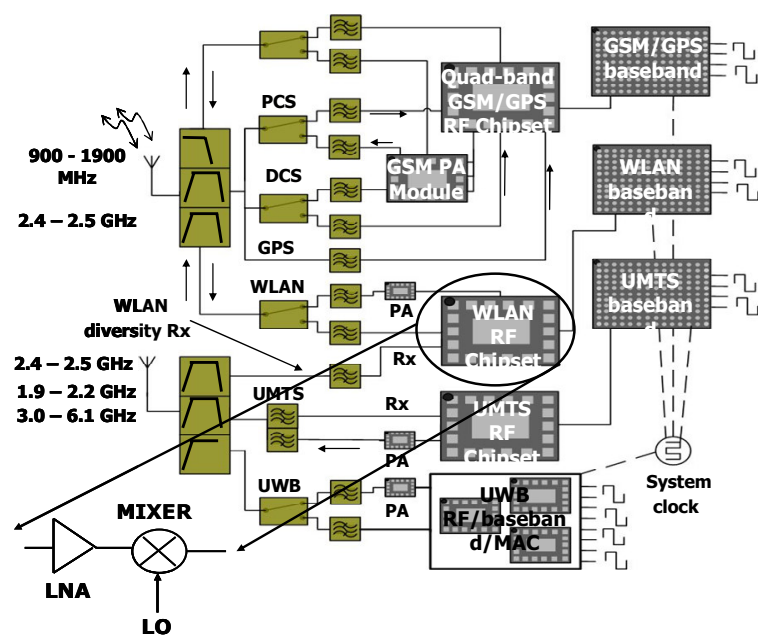

Fig. 1. Multi band RF front end architecture
A multilayer LCP based packaging technology for RF applications is also presented. Baluns using lumped and distributed elements have been designed on this substrate. This approach provides a) high $\mathrm{Q}$ embedded passives using low loss LCP substrate, b) compact size using multiple LCP layers, c) cost reduction because of large panel bench process.

A double balanced ring mixer using these embedded baluns have been fabricated on a 3-layer LCP substrate, for $5 \mathrm{GHz}$ WLAN application. Measurements show 5.1$8.6 \mathrm{~dB}$ conversion loss over $3.5 \mathrm{GHz}$ to $5.5 \mathrm{GHz}$.

\section{LCP SUBSTRATE}

Fig. 2 shows the cross section of the novel multiple LCP layer technology. The new technology incorporates three diclad liquid crystalline polymer layers that are bonded together by a lower melt adhesive. The diclad layers consist of $1 / 2$ ounce copper and one 25 um thick LCP. The LCP dielectric layer has a dielectric constant of 2.95 and a loss tangent of 0.002 . The adhesive layers have a loss tangent of 0.0035 with a dielectric constant of 3.38. The cross-section (Fig.2) shows a six metal layer design where the bottom metal layer is used as a microstrip ground reference for the oscillator. The top metal layer of the cross-section is used for surface mount components and for the incorporation of high Q $(>100)$ inductors. The ability to form micro-vias in the stack up is essential for increased component density as well as for increased routing density. For this application micro-vias were fabricated in the LCP layers to increase component density. The micro-vias were formed using an ultra violet (UV) source. Via diameters of $<100$ um were formed in the LCP layers with extremely high yield and robustness. The LCP process is in fact capable of stacking up to twelve LCP diclad layers which in effect can provide very high component densities of Ls and Cs per unit area.

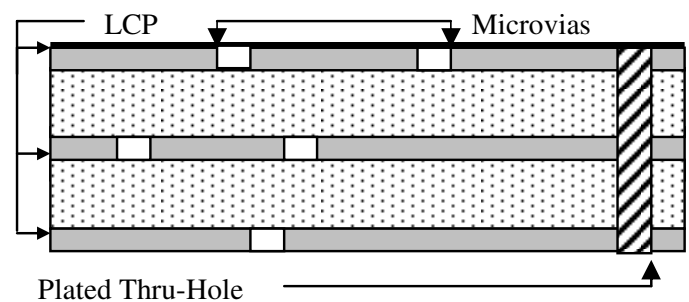

Fig. 2. Cross-section of the M-LCP substrate 


\section{DESIGN OF EMBEDDED BALUNS}

Fig. 3 (a) shows the schematic of a lumped balun composed of two PI networks. Each PI network was designed to transfer a impedance from $50 \Omega$ source to $100 \Omega$ load with $\pm 90^{\circ}$ phase shifts, which result in $180^{\circ}$ difference between the loads. Fig. 3 (b) shows the photograph of the designed lumped balun. The size of the lumped balun is $2.6 \times 1.58 \mathrm{~mm} 2$. Fig. 3 (c), (d) shows the measured results of the designed lumped balun, and tableI summarizes these results.

Balun design using distributed elements (eg. Marchand balun) exhibit wide bandwidths and low-loss, but are also large in size. Several efforts have been published to reduce the size of the Marchand balun using capacitive loading, but small sizes also results in narrow bandwidth. However, [3] has shown the design of compact wide-band baluns using multiband theory. Accordingly, the standard Marchand balun can be made to operate at two different frequency bands by adding lumped reactances in series with the output impedance. The length of the coupled-line segments are chosen to be $\lambda / 4$ at a frequency approximately midway between an upper and a lower band of operation. Neglecting the upper band of operation, the device can be thought of as a single-band balun, but now with an effective electrical length of the coupled line segments considerably lower than $\lambda / 4$ $\left(\theta=90^{\circ}\right)$. A size reduction has thus been achieved without the use of any capacitive loading.

A $50-100 \Omega$ balun operating in the $4.9-5.9 \mathrm{GHz}$ band was designed using this design methodology [3]. Fig. 4 (a) shows the schematic of the balun in planar form, while (b) shows the photograph of the designed Marchand balun. The measured results are shown in (c), (d) and table II , which summarizes the performance of the designed Marchand balun. The fabricated balun measures $3.85 \times 1.34 \mathrm{~mm} 2$, representing a $\sim 64 \%$ size reduction over a conventional Marchand balun.

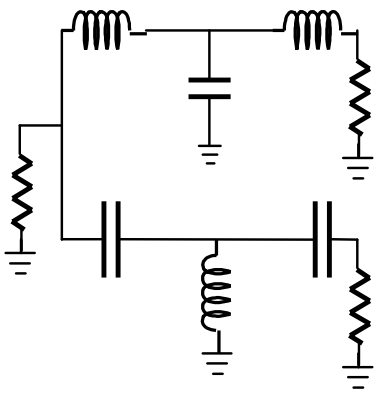

(a)

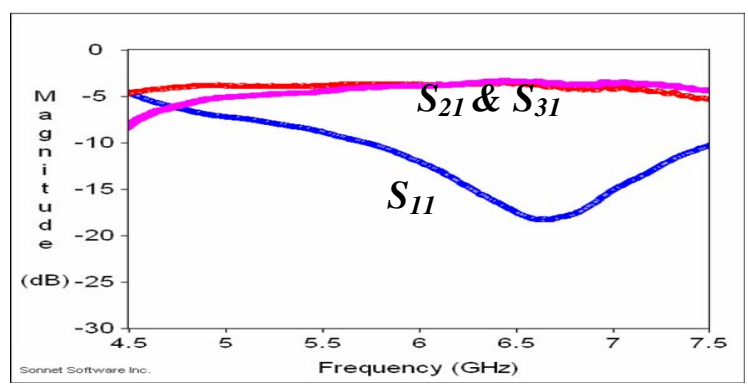

(c)

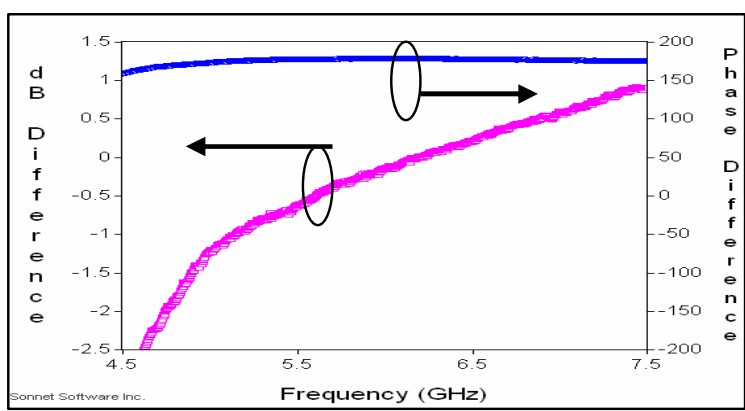

(d)

Fig. 3. Lumped balun. (a) Schematic, (b) Photograph, and (c) Measured results of the lumped balun, and (d) performance summary

\begin{tabular}{|c|c|}
\hline Frequency & $5.8-6.8 \mathrm{GHz}$ \\
\hline Return Loss & $11 \mathrm{~dB}$ \\
\hline Insertion Loss & $0.7 \mathrm{~dB}$ \\
\hline Amplitude Imbalance & $0.5 \mathrm{~dB}$ \\
\hline Phase Imbalance & $3.6^{\circ}$ \\
\hline
\end{tabular}

TABLE 1.

PERFORMANCE OF LUMPED BALUN

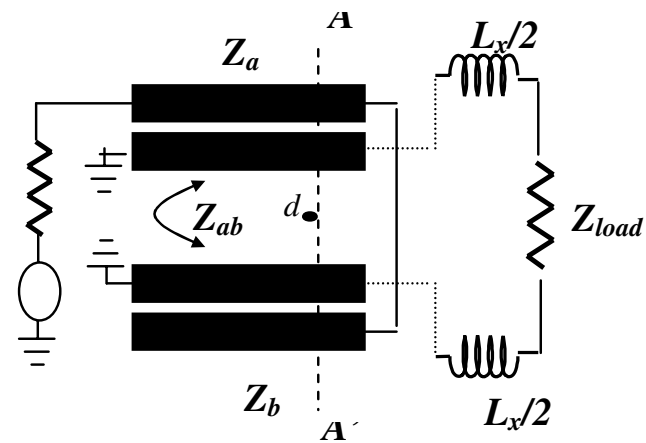

(a)

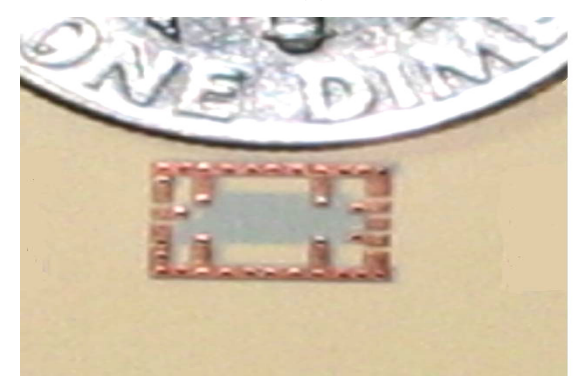

(b)

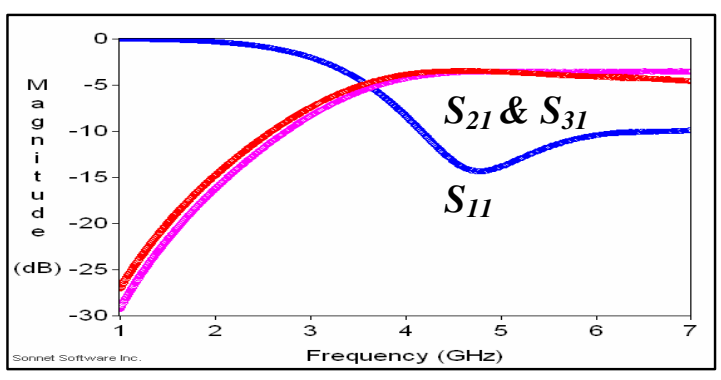

(c) 


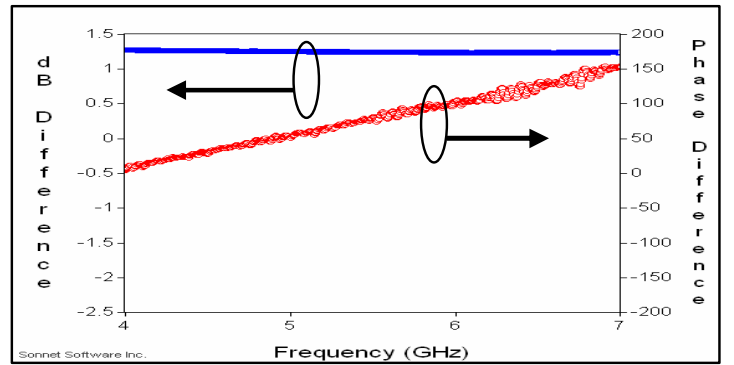

(d)

Fig. 4. Marchand balun. (a) Schematic, (b) Photograph, (c) and (d) Measured results.

\begin{tabular}{|c|c|}
\hline Frequency & $4.9-5.9 \mathrm{GHz}$ \\
\hline Return Loss & $12.3 \mathrm{~dB}$ \\
\hline Insertion Loss & $0.6 \mathrm{~dB}$ \\
\hline Amplitude Imbalance & $0.5 \mathrm{~dB}$ \\
\hline Phase Imbalance & $3^{\circ}$ \\
\hline
\end{tabular}

TABLE 2

PERFORMANCE OF MARCHAND BALUN

\section{DESIGN OF DOUble BALANCED MiXeR}

Fig. 5 shows the schematic of a double balanced ring mixer [4]. The previously designed Marchand balun has been used for RF and IF port. Since the designed balun was 3 ports, it has been modified for 4 port device to accommodate the IF ports. The LO balun was realized using the previously designed lumped balun in the multilayer LCP substrate. Agilent HSMS-8207 silicon Schottky ring diode was surface-mounted, and directly connected to the embedded baluns through vias. The photograph of the fabricated double balance mixer is shown in Fig. 6.

The measured performance of the mixer is shown in fig. 7. Fig. 7 (a) shows the conversion loss with $20 \mathrm{MHz}$ IF bandwidth. The LO power varied from $11 \mathrm{dBm}$ to $13 \mathrm{dBm}$. The conversion loss of $5.1 \mathrm{~dB}$ was achieved at $4 \mathrm{GHz}$, and it ranged from $5.1 \mathrm{~dB}$ to $8.6 \mathrm{~dB}$ over $3.5 \mathrm{GHz}$ to $5.5 \mathrm{GHz}$. Fig. 7 (b) shows the conversion loss with respect to the $\mathrm{RF}$ input power at $4 \mathrm{GHz}$ with $12 \mathrm{dBm}$ of LO power. The conversion loss was approximately $5 \mathrm{~dB}$. Fig. 7 (c) shows the conversion loss variation with respect to IF bandwidth. From DC to $500 \mathrm{MHz}, 8.6 \mathrm{~dB}$ of conversion loss was achieved, and $11 \mathrm{~dB}$ of conversion loss was achieved up to $1200 \mathrm{MHz}$. Fig. 7 (d) shows the isolation of RF to LO and LO to IF.

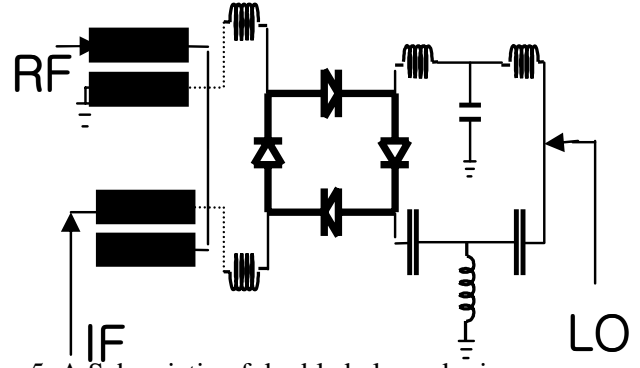

Fig. 5. A Schemiatic of double balanced mixer.

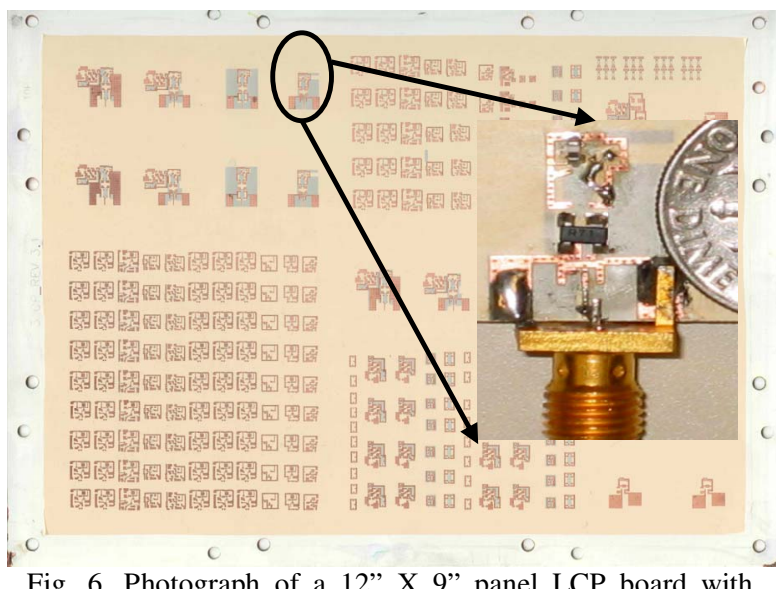

Fig. 6. Photograph of a 12" X 9" panel LCP board with mixer and embedded baluns.

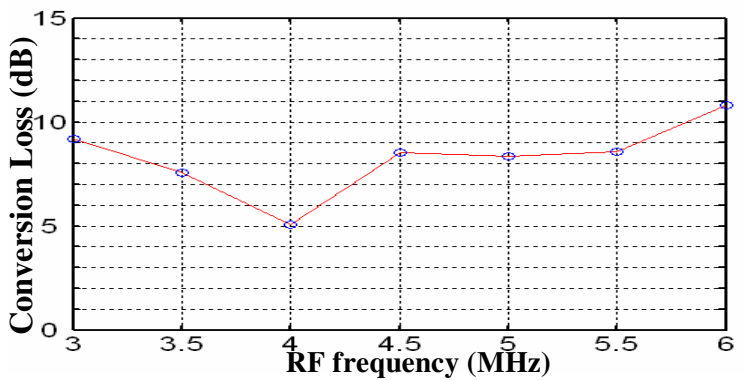

(a)

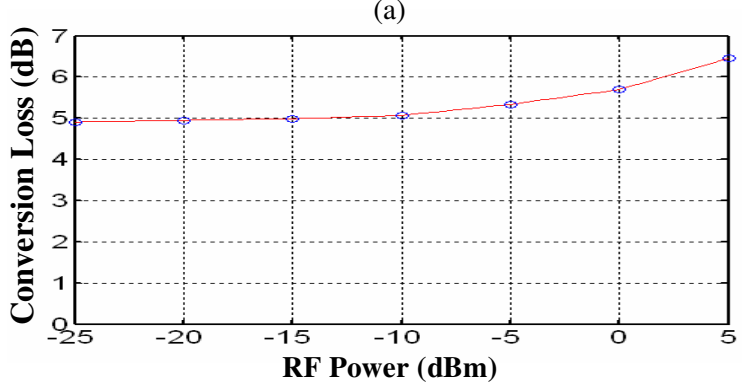

(b)

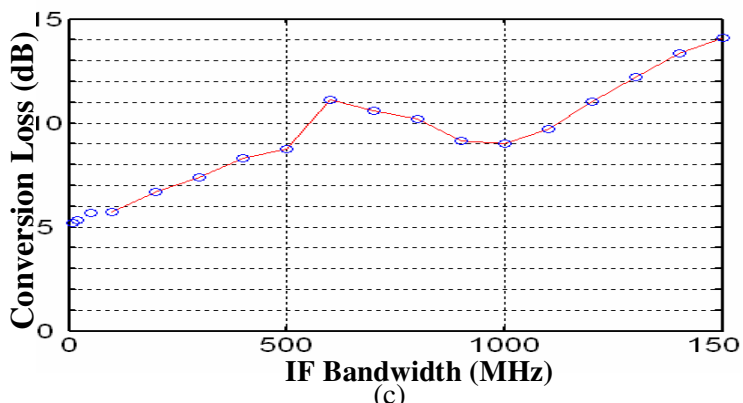

(c) 


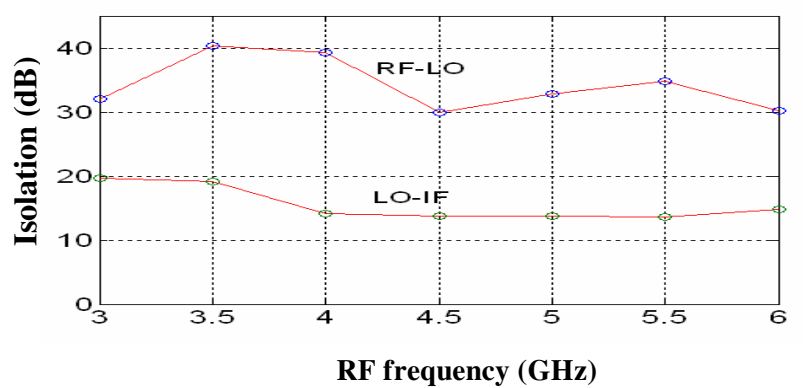

(d)

Fig. 7. (a) Conversion Loss vs RF freq., (b) Conversion loss vs RF power, (c) Conversion Loss vs IF freq., and (d) Isolation between ports

\section{LOW COST MULTILAYER LCP PROCESS}

A novel manufacturing process for multilayer LCP based high quality factor RF Integrated Passive Devices (IPDs), and RF module substrates was demonstrated. This process combines 25 um thick LCP dielectric with low loss tangent glass reinforced organic prepregs in a multilayer stack-up and 4-10 metal layer laminates have been fabricated. For the devices reported in this paper, three LCP layers were circuitized separately, followed by lamination of the LCP layers using organic prepregs. Through holes were mechanically drilled and plated to form interconnections. Liquid photoimageable soldermask was used and electroless nickel immersion gold finish was plated on the bond pads and terminals. All processes including lamination $\left(<200^{\circ} \mathrm{C}\right)$, electroless and electrolytic copper plating and dry film photoresists are compatible with standard FR-4/PWB manufacturing. The panels were fabricated on 12"x18" and 9"x12" format using large area PWB tooling resulting in a lowcost implementation that can be easily scaled to 18 "x 24 " panel size for further cost reduction. Typically ceramic or low temperature co-fired ceramic components are manufactured on substrate sizes less than $8 " \times 8$ ". The fabrication of components on 18"x24" size substrates results in more than a $10 \mathrm{X}$ increase in the number of yielded components for a given board over ceramics. A typical 18"x24" panel can yield more than 5400 devices of $5 \mathrm{~mm} \times 5 \mathrm{~mm}$ size. A top view of the finished laminate is shown in Fig. 6.

The high precision RF passive components in the LCP layers are packaged using the laminate layers providing mechanical strength and enhanced reliability. Unlike conventional PCB materials, this proprietary process technology utilizes organic dielectrics with extremely low moisture uptake comparable to ceramic dielectrics. Typical moisture uptake rates for the packaging materials are $<0.05 \%$ and lead to ceramic-like near-hermetic packaging at organic PCB manufacturing costs. The fully packaged substrate has a CTE matched to typical organic materials used in PWB technology like FR-4 with coefficient of thermal expansion (CTE) around 18$20 \mathrm{ppm} /{ }^{\circ} \mathrm{C}$. The $\mathrm{CTE}$ match allows for large modules to be implemented with very high reliability. The material set can be adjusted to tailorable package CTE in the 3$20 \mathrm{ppm} / \mathrm{C}$ range resulting in expansion matched packages and modules for various RF IC platforms like Si CMOS, $\mathrm{SiGe}$ and GaAs. IC assembly, high frequency electrical/full functional test and over-molding operations are performed on sub-panels of 6"x6" size prior to dicing of the individual modules. A novel and proprietary structure was developed that allows for on-board RF shielding of each of the devices prior to singulation, thereby precluding the need for EMI cans which increases both cost and size. This novel and patented approach results in much lower cost than ceramics with higher performance [5].

\section{CONCLUSION}

A novel 3-layer LCP substrate technology has been used to implement low-loss embedded components for WLAN applications. Using these components, a lumped balun and wideband marchand baluns embedded in the substrate has been realized. Measurements on a lumpeddistributed balun show a percentage bandwidth of $57 \%$ centered around $5.2 \mathrm{GHz}$, for $\mathrm{S} 11<-10 \mathrm{~dB}$, and a size reduction of $64 \%$ over a conventional Marchand balun. A double-balanced ring mixer has been designed using embedded baluns in the multilayer LCP substrate. A lumped balun was used as LO balun and wideband marchand balun was used as RF/IF balun. Measurements on the mixer shows 5.1-8.6 dB conversion loss over 3.5 $\mathrm{GHz}$ to $5.5 \mathrm{GHz}$ with $20 \mathrm{MHz}$ IF bandwidth and $12 \mathrm{dBm}$ of LO power. The results also shows the conversion loss of 5-10 dB with up to $1 \mathrm{GHz}$ IF bandwidth. The RF-LO ports shows more than $30 \mathrm{~dB}$ isolation.

\section{REFERENCES}

[1] P. Pieters, K. Vaesen, G. Carchon, S. Brebels,W. De Raedt, E. Beyne, M.Engels, and I. Bolsens, "Accurate modeling of high-Q spiral inductors in thin-film multilayer technology for wireless telecommunication applications," IEEE Trans. Microwave Theory Tech., vol. 49, pp. 589599, Apr. 2001

[2] S. Dalmia, V. Sundaram, G. White and M. Swaminathan, "Liquid crystalline polymer based RF/wireless components for multi-band applications," Proc. IEEE Electron. Comp. Technol. Conf. (ECTC), pp. 1866-1873, June 2004.

[3] V. Govind, W. Yun, S. Dalmia, V. Sundaram, G. White, M. Swaminathan,"Analysis and Design of Compact Wideband Baluns on Multilayer Liquid Crystalline Polymer (LCP) Based Substrates," Proc. IEEE MTT-S International Microwave Symposium, Long Beach, CA, June 2005.

[4] S.A Maas, Microwave Mixers, $2^{\text {nd }}$ ed. Norwood, MA: Artech House, 1993

[5] George White, Madhavan Swaminathan, Venkatesh Sundaram, Sidharth Dalmia, "Integrated Passive Devices Fabricated Utilizing Multi-layer Organic Laminates", U.S. Patent No. 6,900,708. 